\title{
An Efficient, Economical Slow-Freezing Method for Large-Scale Human Embryonic Stem Cell Banking
}

\author{
Veronique T'Joen, Linde De Grande, Heidi Declercq, and Maria Cornelissen
}

Human embryonic stem cells (hESCs) are one of the most interesting cell types for tissue engineering, cell therapy, basic scientific research, and drug screening. Fast advancement in these areas requires the availability of large amounts of safe and well-characterized hESCs from hESC banks. Therefore, optimized freezing protocols, allowing the cryopreservation of large amounts of hESC without direct contact with liquid nitrogen, need to be established. In this study, 6 different cryoprotector combinations [dimethylsulfoxide (DMSO), ethylene glycol, and hydroxyethylstarch (HES)] combined with 2 different application methods were screened with the VUB01 cell line, to establish a new slow-freezing protocol with high recovery rates and a good expansion capacity. Our best conditions were confirmed in 4 other hESC lines: H1, H9, 181, and UGent2. To our knowledge, this is the first time that HES is evaluated as a cryoprotector for hESCs. The use of $5 \%$ DMSO $+5 \%$ HES combined with a new detachment protocol leads to efficient hESC cryopreservation. This protocol involves treating the hESC colonies with cell dissociation solution, a mild dissociation solution uncommonly used for hESC culture. A recovery ratio ranging from $45.5 \%$ to $168.2 \%$ was obtained, and these were significantly different from the other tested conditions (Student's $t$-test, $P<0.05$ ). The cryopreserved hESCs were morphologically comparable to control cells, exhibited a good expansion profile, were positive for pluripotent expression markers, and could still differentiate into the 3 germ layers. This new protocol allows efficient and economical hESC cryopreservation, ideal for hESC banking.

\section{Introduction}

Since the First derivation of human embryonic stem cells (hESCs) [1], scientists have been researching these cells and discovering their importance, capabilities, and complexity. These unspecialized cells are capable of long-term self-renewal in vitro while retaining their capacity to differentiate into all cell types from the 3 germ layers, an ability called "pluripotency." These remarkable cell characteristics turn hESCs into one of the most interesting cell types for tissue engineering purposes, advancements in drug screening, discoveries in basic scientific research, and the conduction of developmental studies [2,3]. One prerequisite for fast progression in these areas is the availability of large amounts of well-characterized hESCs from hESC banks. The banking of hESCs for research and future clinical applications requires optimized cryopreservation and thawing protocols.

Currently, 2 techniques are applied for the cryopreservation of hESCs: vitrification and slow freezing-rapid thawing. Vitrification is favored by several groups for the cryopreservation of hESCs [4-7]. Using open pulled straw methods, survival rates of $70 \%-90 \%$ have been reported. However, there are several disadvantages preventing the widespread use of this technique. First, high concentrations of cryoprotectors, which are cytotoxic above $4^{\circ} \mathrm{C}$, are needed to rapidly obtain the solidified glass-like state $[8,9]$. Second, the procedure requires serial dilutions in different cryoprotector media, which must be performed fast and precisely. Consequently, this task needs to be performed by trained specialists and is, in itself, very labor intensive. Additionally, vitrification is also mostly performed in open pulled straws. In the open straw, contact between the liquid nitrogen and the cells is established, which carries the risk of bacterial and fungal contaminations [10]. A major disadvantage, however, is that only a low amount of hESC colonies can be vitrified per run. Hence, scaling-up the experiment to freeze bulk quantities of hESCs poses inherent problems.

In contrast, during the slow-freezing procedure, only low amounts of cryoprotector are added to the solution, no serial dilutions or laborious tasks need to be performed, and higher amounts of hESC s can be frozen in 1 run in a closed cryovial. The major difficulty in slow freezing is determining the right cryoprotector combination and concentration to adequately stabilize the cells and the ice nucleation during the freezing 
process. Many scientists have looked into slow-freezing protocols for hESCs as an alternative for vitrification [11-14]. Dimethylsulfoxide (DMSO) is the most popular cryoprotector for hESC slow freezing, often in combination with fetal bovine serum (FBS) and ethylene glycol (EG). Other scientists are exploring the cryopreservation of adherent hESCs $[5,15]$. Recently, most cryopreservation protocols focus on the addition of a Rock inhibitor (Y-27632) during the different stages of the freezing process as a means to ameliorate the recovery rate of the hESCs [16-20].

In this article, 6 different cryoprotector combinations of DMSO, hydroxyethylstarch (HES), and EG [14,21,22] were tested. Cryoprotectors are added during the freezing process to have a better control over the ice formation and cell damage that occurs because of the accumulation of intracellular and extracellular ice crystals. There are 2 types of cryoprotectors-intracellular and extracellular cryoprotectorsboth with a different impact on the freezing process. Intracellular cryoprotectors, such as DMSO, EG, and glycerol, have a low molecular weight and thus can penetrate the cell membrane and gradually substitute the $\mathrm{H}_{2} \mathrm{O}$ present in the cells. The osmolality of the cells is thereby increased, and subsequently, the percentage of extracellular $\mathrm{H}_{2} \mathrm{O}$ that can form ice crystals before reaching the osmotic equilibrium is reduced and total dehydration of the cells is prevented [8,10,23-25]. Extracellular cryoprotectors, such as polyvinyl pyrrolidone, HES, polyethylene glycol, dextran, trehalose, and sucrose, cannot penetrate the cell membrane and stabilize the cell by forming a viscous glassy shell around its surface $[8,25]$. By combining intracellular and extracellular cryoprotectors, both the inner cell and the cell surrounding is stabilized, which in theory should lead to a better cell survival once an ideal combination is found.

These cryoprotectors were tested in combination with 2 application methods and an optimized thawing protocol. Our aim was to find an efficient cryopreservation method with a high recovery ratio that allows freezing of large amounts of hESCs while retaining their pluripotency and expansion capacity.

\section{Materials and Methods}

\section{Feeder preparation: mouse embryonic fibroblasts}

Mouse embryonic fibroblasts (MEFs) originating from MF1 mouse embryos between passage 0 and 4 were used as a feeder layer to sustain the hESC culture. The MEFs were treated with $10 \mu \mathrm{g} / \mathrm{mL}$ mitomycin C (Sigma Aldrich) for $3 \mathrm{~h}$ to arrest mitosis and seeded at a density of 8,500 cells $/ \mathrm{cm}^{2}$ on gelatin-coated tissue culture plates.

\section{hESC standard culture protocol}

The hESC lines used in these experiments were the VUB01 [26] (VUB), H1 (Wicell), H9 (Wicell), 181 (Karolinska Institute), and UGent2 hESC line [27] (Department for Reproductive Medicine, Ghent University Hospital, Gent, Belgium). The hESCs were cultured on mitomycin-C inactivated MEFs on tissue culture plates at $37^{\circ} \mathrm{C}$ under $5 \% \mathrm{CO}_{2}$. The hESC medium consisted of 80\% Dulbecco's modified Eagle's medium (DMEM)-F12 (Invitrogen) $+20 \%$ serum replacement (Invitrogen) $+1 \mathrm{mM}$ L-glutamine (Invitrogen) $+0.1 \mathrm{mM}$ nonessential amino acids (NEAA) (Invitrogen) $+0.1 \mathrm{mM} \beta$-mercapto-ethanol
(Invitrogen) $+4 \mathrm{ng} / \mathrm{mL}$ basic fibroblast growth factor) (Millipore). The medium was changed daily and the cells grew as dense colonies. Weekly, the hESC colonies were split by microdissection using sharp glass-cutting pipettes.

\section{hESC slow-freezing protocols}

A large-scale slow-freezing setup was performed with the VUB01 cell line. Two detachment protocols were applied to obtain pure hESCs. In protocol 1, the hESC colonies were detached as entire colonies with collagenase IV. In protocol 2, the hESC colonies were detached with collagenase IV and subsequently treated with a cell dissociation solution for $5 \mathrm{~min}$. Six different cryopreservation solutions were tested: $10 \%$ DMSO + 90\% HM, 5\% DMSO + 5\% HES + 90\% HM, 10\% $\mathrm{DMSO}+10 \% \quad \mathrm{HES}+80 \% \quad \mathrm{HM}, \quad 10 \% \quad \mathrm{EG}+90 \% \quad \mathrm{HM}, \quad 5 \%$ $\mathrm{EG}+5 \% \mathrm{HES}+90 \% \mathrm{HM}$, and 10\% EG + 10\% HES + 80\% HM. After preparation, $1 \mathrm{~mL}$ of the cryosolution with cells was transferred to a cryovial (Cryo.S ${ }^{\mathrm{TM}}$; Greiner Bio-One), cooled slowly (cooling rate $-1.5^{\circ} \mathrm{C} / \mathrm{min}$ ) to $-80^{\circ} \mathrm{C}$, and then stored in a liquid $\mathrm{LN}_{2}$ storage device. $\mathrm{HM}$ medium consisted of $80 \%$ DMEM-F12 (Invitrogen) $+20 \%$ serum replacement (Invitrogen) $+400 \mu \mathrm{L}$ HEPES. The best conditions were repeated with the H1, H9, 181, and UGent2 cell line.

\section{Thawing of hESCs}

All experimental slow-freezing protocols were thawed in a water bath of $37^{\circ} \mathrm{C}$, until an icy mass remained. Sucrose $(0.1 \mathrm{M})-\mathrm{HM}$ medium was added and incubated for $5 \mathrm{~min}$. Then the cell suspension was centrifuged and plated out onto fresh MF1 feeders.

\section{Evaluation after thawing and data collection}

All experiments were repeated in triplicate, and all vials were kept for minimally 1 week in $\mathrm{LN}_{2}$. The amount of hESC colonies, obtained from full-grown plates, was counted before freezing, after thawing, and at 7 days of culture postthawing. The hESC colonies were morphologically evaluated and counted using a gradual scoring system $[5,28]$ on an inverted phase-contrast light microscope with objective $4 \times$ (Olympus inverted Research System Microscope IX81). For each counted colony number, an uncertainty degree of $5 \%$ was taken into account. This degree of uncertainty was established after repeated counting experiments of the same plate and used to determine the error bars in the statistical graphical representations [29]. Only grade A (>80\% undifferentiated) and grade B (between 50\% and 80\% undifferentiated) colonies were selected for further use in the evaluation as these are the only colonies capable of generating new pluripotent colonies in the next passage. Therefore, the recovery rate was calculated as follows: the amount of Grade $\mathrm{A}+\mathrm{B}$ colonies at day 7 postthawing versus the amount of frozen Grade A+B colonies. The Student's t-test was used for determining significant differences between the obtained amounts of colonies after freezing. A $P$ value of $<0.05$ was considered significant.

\section{hESC: expansion profile}

The thawed hESCs were followed for a minimum of 3 passages, to evaluate changes in their expansive behavior. By 
use of the same counting and gradual scoring system, as mentioned earlier, combined with the known split ratio, the total amount of colonies was calculated for each passage. The mean of the total amount of colonies (experiments performed in triplicate) was plotted.

\section{hESC: size analysis}

For size analysis, digital images were recorded during the cryopreservation experiments. A minimum of 100 randomly selected hESC colonies or clumps were measured using the Excellence $^{\circledR}$ software of the Olympus microscope system (Olympus Inverted Research System Microscope IX81, brightfield, objective $4 \times$ ). Mann-Whitney test was performed to evaluate statistical differences. A $P$ value of $<0.05$ was considered significant.

\section{Immunocytochemical analysis of hESC colonies}

hESC pluripotency was determined at passage 5 by immunostaining for SSEA-3, SSEA-4, TRA 1-60, TRA 1-81, and Oct4 (isoform 4A) (antibodies from Santa Cruz Biotechnology, Inc.) and by alkaline phosphatase staining (BCIP/NBT Liquid Substrate System by Sigma). For the immunostaining, colonies were fixed in $1 \%$ paraformaldehyde for $10 \mathrm{~min}$, washed with phosphate-buffered saline (PBS), and blocked with blocking serum (PBS/5\% normal rabbit or goat serum/ $0.2 \%$ Tween) for $30 \mathrm{~min}$. Primary antibodies used were rat monoclonal anti-SSEA-3 (IgM 1/200), mouse monoclonal anti-SSEA-4 (IgG 1/500), mouse monoclonal anti-TRA 1-60 (IgM 1/100), mouse monoclonal anti-TRA 1-81 (IgM 1/50), and mouse monoclonal anti-Oct4 (IgG 1/100). After $1 \mathrm{~h}$ of incubation, the cells were washed with PBS and incubated with the secondary antibody (FITC-labeled goat anti-mouse IgM $1 / 500$ or biotin labeled rabbit anti-mouse IgG) for $30 \mathrm{~min}$. In case a biotin-labeled secondary antibody was used, visualization was achieved with streptavidin-FITC coupling (30 min). After washing, the cells were covered with PBS and evaluated by fluorescence microscopy (Olympus inverted Research System Microscope IX81, GFP filter, Cell ${ }^{\mathrm{M}}$ software).

\section{hESC embryoid body formation}

Plates with full-grown hESC colonies were selected. The hESCs were detached from the feeder layer by incubation with collagenase IV solution $(1 \mathrm{mg} / \mathrm{mL})$ for $30-40 \mathrm{~min}$. The colonies were pelleted by centrifugation for $3 \mathrm{~min}$ at $90 \mathrm{~g}$, resuspended in embryoid body (EB) medium, and transferred to shaker flasks. The EB medium consisted of $80 \%$ DMEM-F12 (Invitrogen) $+20 \%$ FBS (Invitrogen) $+1 \mathrm{mM} \mathrm{L-}$ glutamine (Invitrogen) $+0.1 \mathrm{mM}$ NEAA (Invitrogen) and $0.1 \mathrm{mM} \beta$-mercapto-ethanol (Invitrogen). EBs were formed at passage 10 and cultured for 21 days in these settings.

\section{Real-time RT-PCR analysis of hESC colonies and $E B s$}

The colonies were detached from the feeder layer using collagenase IV solution ( $1 \mathrm{mg} / \mathrm{mL}$; Invitrogen) for 30-40 min, collected, and centrifuged at $90 \mathrm{~g}$ for $3 \mathrm{~min}$. After removal of the supernatant, TRI reagent was added to obtain lysis of the cells. The RNA was isolated using chloroform and further purified with isopropanol and ethanol. Afterward, DNA treatment was performed with a DNase kit (Invitrogen) and the RNA content and purity was examined by spectrophotometry. The RNA was transcribed to cDNA using the Reverse Transcriptase Core Kit (Eurogentec) on a Thermocycler (Applied Biosystems 2720 Thermocycler). Real-time PCR was performed on the ABI 7500 Fast Real-Time PCR device with ABI Taqman probes (Applied Biosystems) for the following genes: OCT4 (Hs00732896_s1), NANOG (Hs02387400_g1), HAND1 (Hs00231848_m1), COL2A1 (Hs01064869_m1)， CD34 (Hs00990732_m1), RUNX2 (Hs00231692_m1), NESTIN (Hs00707120_s1), SOX9 (Hs00165814_m1), PAX6 (Hs01088112_m1), CERBERUS (Hs00193796_m1), AFP (Hs00173490_m1), and GATA4 (Hs00171403_m1). GAPDH was selected as endogenous control. Analysis of the gene expression was done using the ABI software for gene expression analysis on the ABI 7500 Fast Real-Time device.

\section{Results}

The VUB01 cell line was selected for a large-scale slowfreezing study. Figure 1 shows a comparison of the mean recovery rates of the different experimental conditions. When protocol 1 was applied, a successful startup of the colonies was obtained in all conditions with DMSO.

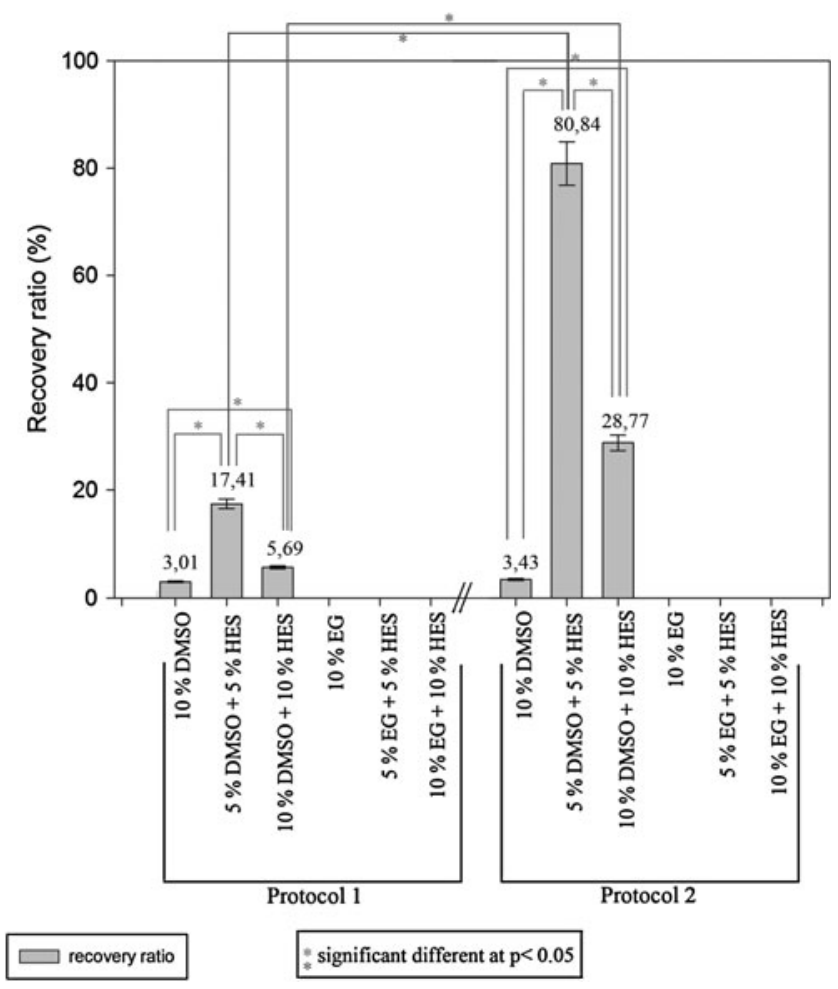

FIG. 1. Comparison of the recovery ratios at day 7 postthawing of the VUB01 cell line under different experimental conditions. Application of protocol 1 led to a successful startup of the colonies for all conditions containing dimethylsulfoxide (DMSO). The best recovery rate was obtained with 5\% DMSO and 5\% hydroxyethylstarch (HES). Similar results were obtained with protocol 2; however, the recovery rates are much higher. The use of ethylene glycol (EG) was unsuccessful. 
Combination of DMSO with HES had a beneficial effect on the recovery rate. Especially, the experimental conditions with 5\% DMSO + 5\% HES and 10\% DMSO +10\% HES had good recovery rates $(17.41 \% \pm 0.87 \%$ and $5.69 \% \pm 0.28 \%)$. Successful conditions were not established with the use of EG alone or with the addition of HES. Our results $(10 \%$ DMSO, 5\% DMSO + 5\% HES, and 10\% DMSO+10\% HES) are significantly different from each other (2-tailed Student's $t$-test, $P<0.05)$. A similar profile in the recovery rates can be seen after application of protocol 2. Again, only conditions with DMSO in the cryopreservation solution resulted in successful plates. However, application of the second protocol led to much higher recovery rates compared with application of protocol 1 . Recovery rates of $80.83 \% \pm 4.04 \%$ for the condition with 5\% DMSO +5\% HES and $28.77 \% \pm 1.44 \%$ for the condition with 10\% DMSO $+10 \%$ HES were obtained. For these 2 conditions, this equals a 5 -fold increase in recovery ratio. Compared with the results of protocol $1(10 \%$ DMSO, 5\% DMSO + 5\% HES, and 10\% DMSO+10\% HES), we again obtained significant different values (2-tailed Student's $t$-test, $P<0.05)$. Additionally, all cryopreserved samples with 5\% DMSO +5\% HES and 10\% DMSO +10\% HES were successfully thawed. The use of $10 \%$ DMSO not always led to a successful startup (failure of 1 in 3 samples).

Figure 2 depicts the expansion profile of the hESCs during 3 passages, which were cryopreserved under different experimental conditions. For both protocols, an unfrozen control sample was included as a reference. The best expansion profile using protocol 1 was obtained with 5\% DMSO $+5 \%$

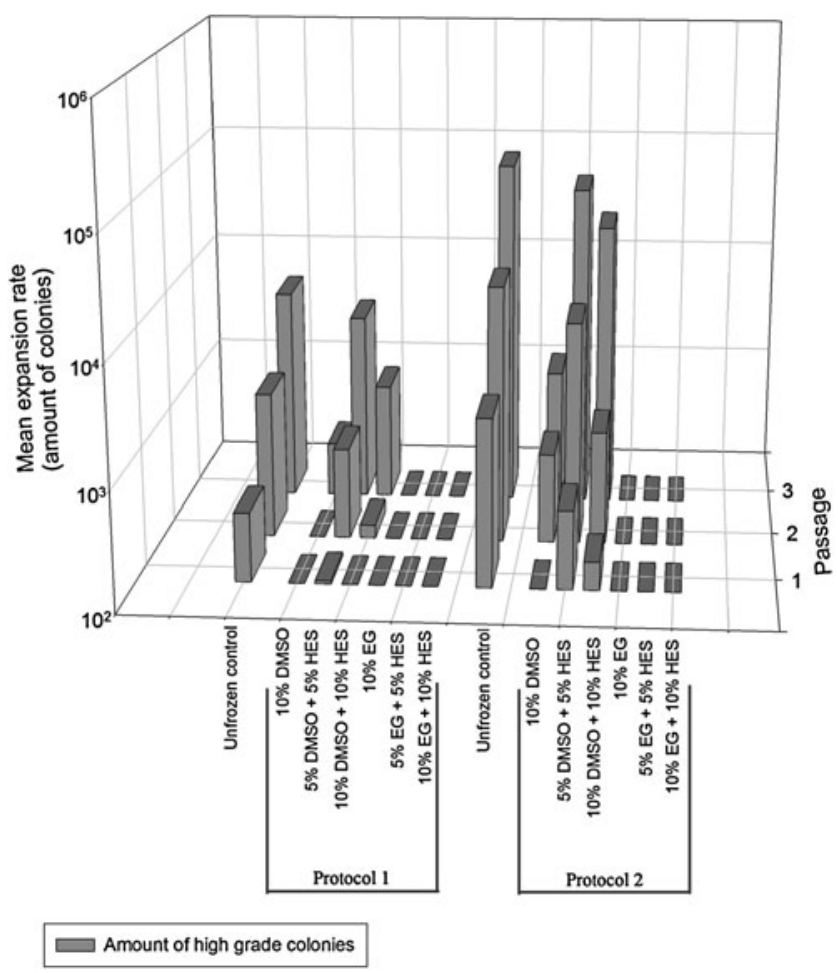

FIG. 2. Expansion data of the VUB01 cell line, after cryopreservation under the different experimental conditions. The best expansion profile of the human embryonic stem cells (hESCs) after freezing can be seen in the condition with $5 \%$ DMSO and 5\% HES. Application of protocol 2 leads to a better expansion than application of protocol 1.
HES. Here, a mean amount of 3,980 \pm 199 colonies was obtained after 3 passages. With the unfrozen control sample, a mean amount of $6,368 \pm 318$ colonies was obtained. The expansion profile of the colonies, cryopreserved with protocol 2 , is again better than those cryopreserved with protocol 1 . After 3 passages, the mean amount of colonies was $56,910 \pm 2,845$ for the condition with $5 \%$ DMSO $+5 \%$ HES. With the unfrozen control sample, a mean amount of $89,712 \pm 4,486$ colonies was obtained.

The pluripotency of the VUB01 cells cryopreserved using the best condition (protocol 2, 5\% DMSO +5\% HES $+90 \%$ $\mathrm{HM}$ ) was checked with immunostaining and is shown in Fig. 3. The colonies were positive for SSEA-3, SSEA-4, TRA 1-60, TRA 1-81, and Oct4. The detailed immunofluorescence pictures clearly show a surface localization of SSEA-3, SSEA-4, TRA 1-60, and TRA 1-81. The Oct4 staining (isoform A) shows a general nuclear localization. The colonies also expressed high amounts of alkaline phosphatase.

Additionally, real-time PCR analysis was performed on cultured hESCs, on previously frozen and thawed hESCs (day 7 postthawing), and on their derived EBs to confirm the results of the immunostaining and to examine the differentiation capacity of the hESCs after freezing. The relative quantitative gene expression profiles can be found in Fig. 4. The frozen/thawed hESCs show an equally high, even a slightly increased expression of Oct 4 and Nanog when compared with the control hESCs. This indicates that there is no decrease in colony quality related to the cryopreservation. The derived EBs showed a decrease in expression of Oct4 and Nanog and an increase of several differentiation markers. Upregulation of Sox9 and Pax6 indicates that differentiation is occurring in the ectodermal lineage. The expression of Nestin is virtually the same as in the control sample. Upregulation of Hand1, Col2A1, CD34, and Runx2 indicates differentiation along the mesodermal lineage. Upregulation of AFP and GATA4 indicates differentiation along the endodermal lineage. Cer1 is downregulated at this stage.

The best conditions, cryopreservation with 5\% DMSO+ $5 \% \mathrm{HES}+90 \% \mathrm{HM}$ and 10\% DMSO + 10\% HES + 80\% HM, were repeated with 4 other hESC lines (Fig. 5) to verify the reproducibility. For all cell lines, similar results were obtained. The application of protocol 2 is consistently better than the application of protocol 1 and the highest recovery rates are obtained with 5\% DMSO +5\% HES + 90\% HM.

The size of the cryopreserved hESC colonies was also examined, as this is an important factor in the cryopreservation process. Figure 6 shows an overview of the colony size at cryopreservation. Colonies frozen with protocol 1 have a mean size of $0.167 \pm 0.008 \mathrm{~mm}^{2}$ and those frozen with protocol 2 measure $0.0052 \pm 0.0002 \mathrm{~mm}^{2}$. Statistical analysis (Mann-Whitney test, $P<0.05$ ) revealed that these differences are significant. Additionally, the size distribution of the colonies frozen with protocol 2 is much more narrow than those frozen with protocol 1. Less variation and more uniformity in clump size were obtained using the second protocol. This can be deducted from the box-plot data and is confirmed by the illustrative phase-contrast microscopy pictures.

\section{Discussion}

The aim of the present study was to investigate several slow-freezing-rapid-thawing protocols for hESCs enabling 

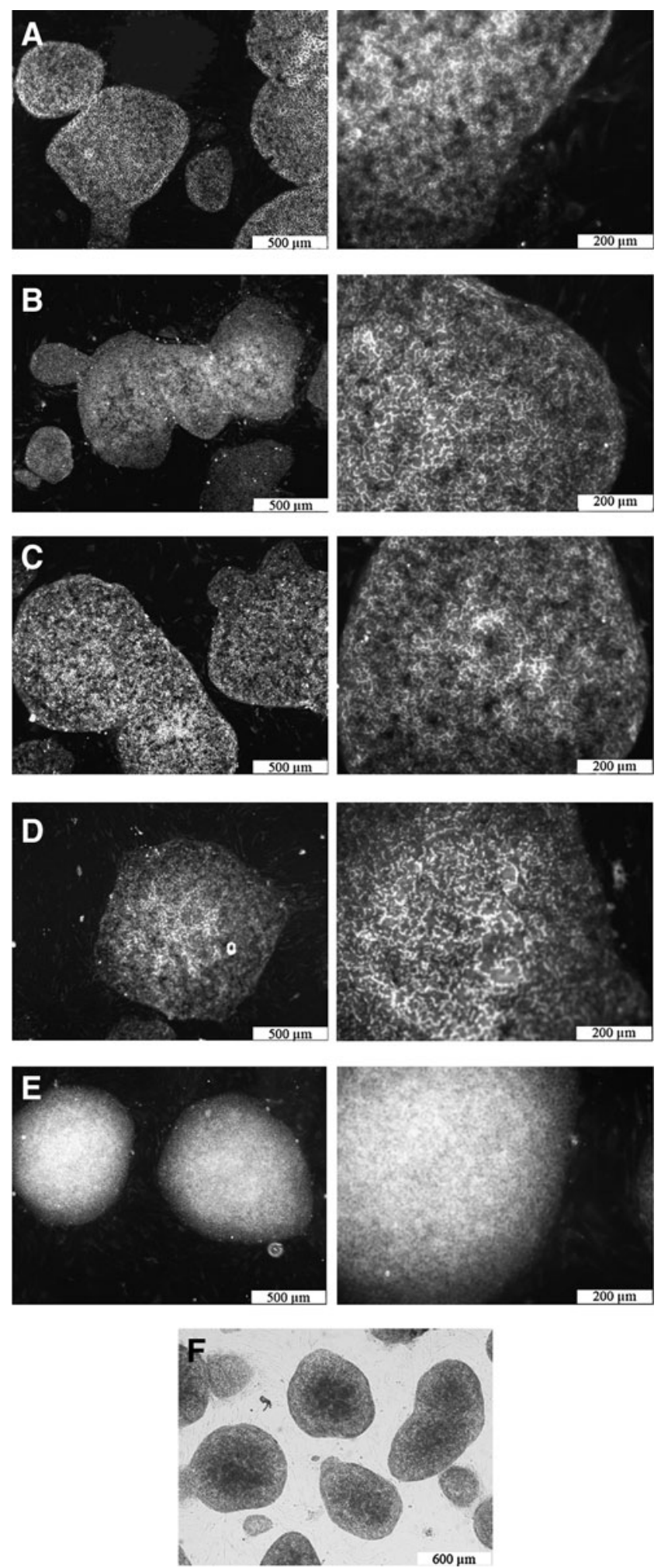

FIG. 3. Pluripotency evaluation of the VUB01 hESC line, frozen with the adapted protocol and 5\% DMSO +5\% HES + $90 \%$ HM. Positive stainings were obtained for all markers. In the overview image, but more specifically in the detailed images of SSEA-3, SSEA-4, TRA 1-60, and TRA 1-81, it is clear that the expression of the markers is localized on the surface, as anticipated. Immunostaining for Oct4 (Isoform 4A) shows a broad nuclear localization. (A) SSEA-3; (B) SSEA-4; (C) TRA 160; (D) TRA 1-81; (E) Oct4; (F) alkaline phosphatase staining.

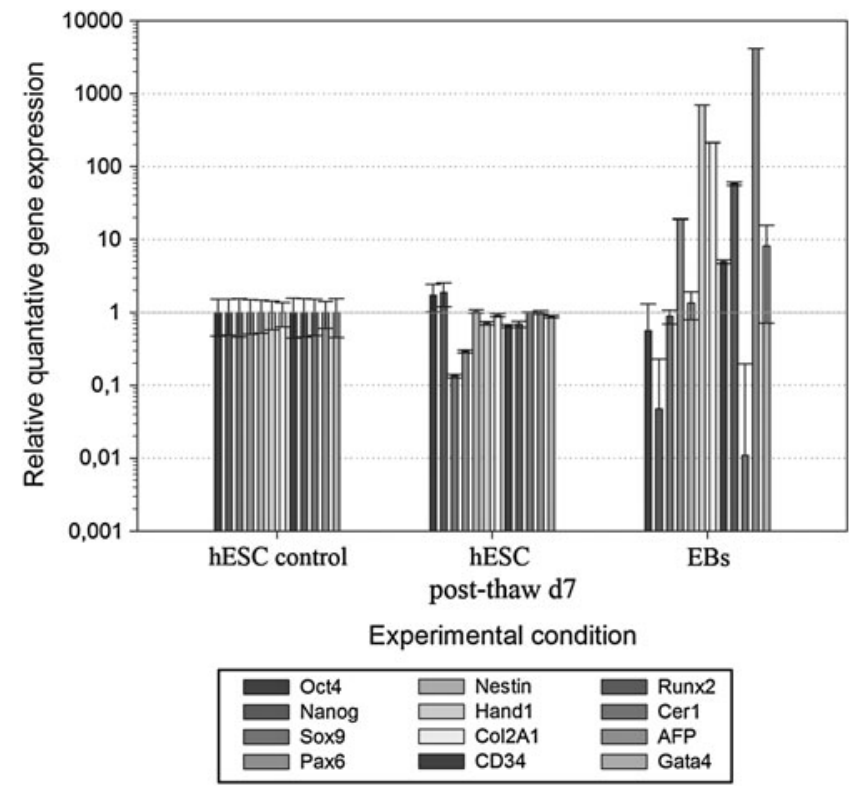

FIG. 4. Real-time PCR data regarding pluripotency and differentiation capacity of the VUB01 cell line, frozen with protocol 2 and 5\% DMSO +5\% HES $+90 \% \mathrm{HM}$. The relative quantitative gene expression profile of 3 datasets [hESC control, hESCs at day 7 postthawing, and derived embryoid bodies (EBs)] are compared with each other. There is a high expression of Oct 4 and Nanog in the thawed hESCs, slightly higher than in the control hESCs. Additionally, there is no upregulation of differentiation markers visible. The EBs show a decrease in Oct 4 and Nanog expression, indicating that differentiation is occurring. Moreover, there is an increase in several differentiation markers, characteristic of the 3 germ layers.

the cryopreservation of large amounts of hESCs without direct liquid nitrogen contact. Although vitrification is commonly used for the cryopreservation of hESCs and high survival rates are reported, we decided to focus on slow freezing in the perspective of large-scale hESC banking. The inherent high technical requirements of vitrification and, especially, the need to work with very small volumes to achieve good results make large-scale vitrification difficult. However, a new method for bulk vitrification of hESCs was recently suggested by $\mathrm{Li}$ et al. [4,5], using a vitrification vial with a steel mesh instead of the commonly used open straws. Nevertheless, as the cryopreservation is performed in open vials, an increased risk of contamination and cell infection is present [30].

In the present study, we attempted to eliminate high amounts of cryoprotectors, serum, or other added substances and to reduce the risk of contamination during freezing by the use of closed cryovials. By combining intracellular and extracellular cryoprotectors, both the inner cell and the cell surrounding are stabilized, which in theory should lead to a better cell survival once an ideal combination is found. To our knowledge, this is the first time that HES is evaluated as a cryoprotector in hESC culture systems. HES, in combination with DMSO, is widely used for the cryopreservation of cord blood, bone marrow, and hematopoietic stem cells $[14,21,22]$. For these cell types, it has 


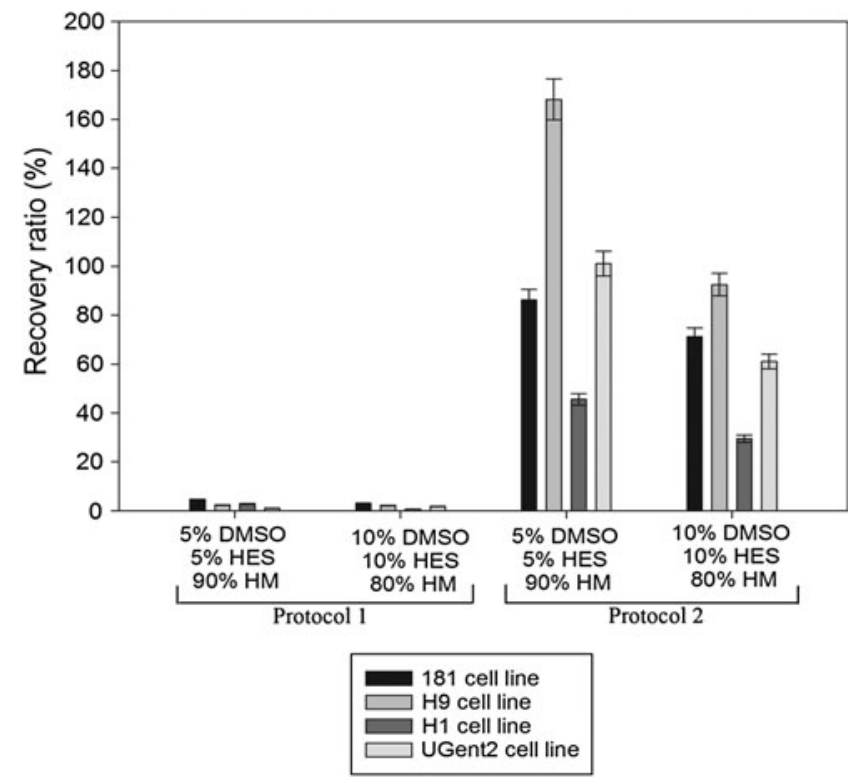

FIG. 5. Overview of the hESC recovery ratio of 4 different hESC lines. The highest recovery ratios are consistently obtained with the application of protocol 2 (5\% DMSO and 5\% HES).

been proven to be beneficial to use the combination of $5 \%$ DMSO and $6 \%$ HES instead of $10 \%$ DMSO. It results in a higher nucleated cell recovery, a better cell viability, and a higher burst-forming (BFU-E) and colony-forming (CFUGM) potential [14]. From a clinical point of view, this reduced DMSO protocol also led to less clumping of the cells at infusion, combined with a reduced incidence and severity of transfusion-related reactions and improved kinetics of engraftment. These observations were made with mesenchymal and hematopoietic stem cells, and as such, it is interesting to investigate the effect of this cryoprotector on embryonic stem cells.

We compared the recovery/survival rates of the colonies detached before freezing either by collagenase IV alone (protocol 1) or by collagenase IV combined with the cell dissociation solution (protocol 2). The recovery rate was calculated at day 7 postthawing/postplating. At this time point, the mean colony size of previously frozen and unfrozen control colonies is comparable and morphological gradual scoring could be easily performed. In general, for both protocols, 5\% DMSO +5\% HES + 90\% HM turned out to be the best solution. However, the recovery rate was much higher and also significantly different $(P<0.05)$ when protocol 2 was applied. This was confirmed in all 5 cell lines tested.

Analysis of the cryopreservation data using protocol 1 or 2 showed that slow freezing with DMSO was always successful. The use of EG had a negative impact on our cryopreservation protocols. Similar results were obtained by Ha et al. [12]. Their study clearly showed that only 5\% DMSO in combination with high amounts of FBS (95\%) allowed hESCs to survive the cryopreservation process, although at a low recovery rate. However, this protocol could be ameliorated by combining $5 \%$ DMSO with $10 \%$ EG and only $50 \%$ FBS. Ha

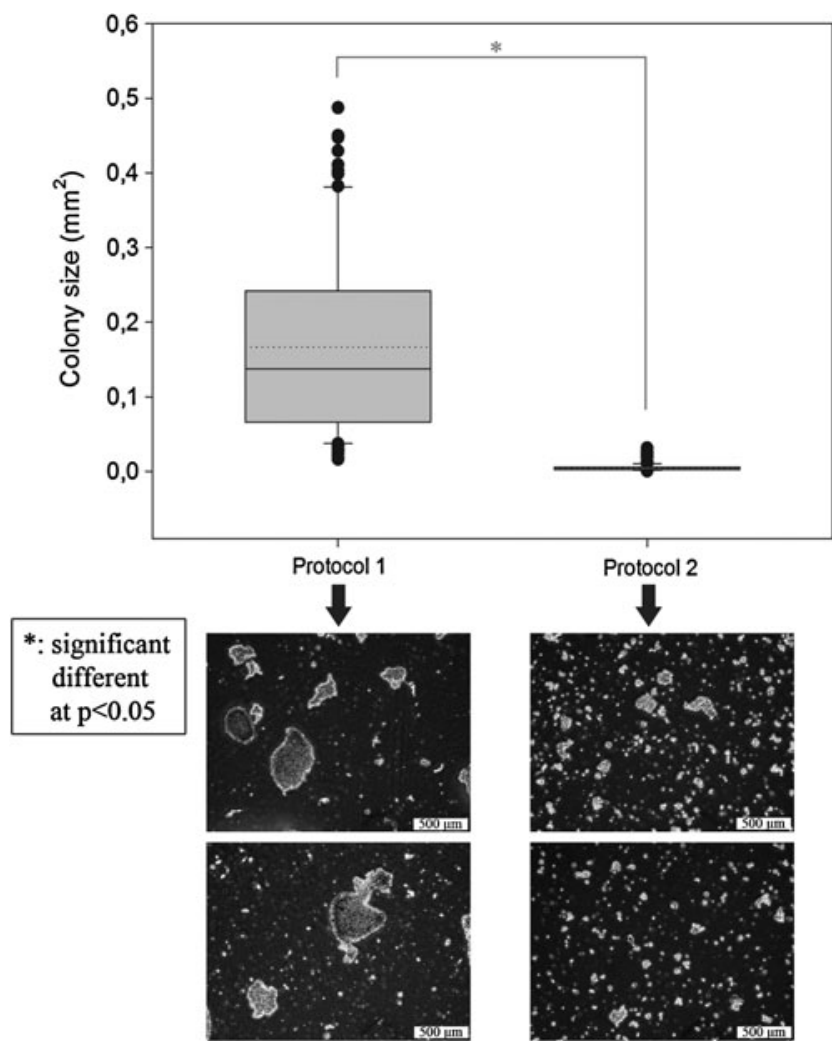

FIG. 6. Colony size distribution at cryopreservation. Colonies frozen with protocol 2 are much smaller than those frozen with protocol 1, as can be deducted from the box-plot data and confirmed by the illustrative phase-contrast microscopy pictures. Additionally, the hESC clumps are more uniform in size and show less variation.

et al. were able to obtain a survival rate of $30.2 \%$ and also observed low recovery rates with EG. However, for our objective, the use of high amounts of FBS would be inacceptable. In our protocol, an HM medium, which contains only $20 \%$ knock-out serum replacement (also known as synthetic serum), is applied.

In protocol 2, the hESCs were additionally treated with the cell dissociation solution before freezing. The cell dissociation solution is a very mild, nonenzymatic, animal-free solution used for cell passaging. It has been previously tested in combination with hESCs for single-cell passaging with positive effects [31]. The application of the cell dissociation solution before freezing reduces the mean hESC clump size. Moreover, the clump size is more uniform, thereby creating a mixed population of very small hESC clumps and single cells before freezing. Subsequently, the penetration of DMSO into the hESCs and the extracellular stabilization by HES should more easily occur, thus allowing a faster/better installation of the osmotic equilibrium and less ice damage. This was confirmed by our results for all tested cell lines. The combination of $5 \%$ DMSO $+5 \%$ HES and $10 \%$ DMSO $+10 \%$ HES resulted in higher recovery rates, compared with the single use of DMSO. Other scientists have also investigated freezing of small clumps and single-cell hESCs [16,18,32,33]. Combinations of $10 \%$ DMSO with high amounts of synthetic serum or $10 \%$ DMSO with hESC medium and Rock inhibitor 
(Y-27632) generally led to good results. Often TrypLE ${ }^{\mathrm{TM}}$ Express or trypsin/EDTA was used as a dissociative agent and the Rock inhibitor (mostly $10 \mu \mathrm{M}$ ) could be added before, during, and after the cryopreservation process. Without the use of Y-27632, the results were rather poor. It has been postulated that Y-27632 acts by desensitizing the single cells to go into anoikis upon dissociation. The exact reaction mechanism of Y-27632 is, at the moment, unknown and a lot of crosstalks between pathways occur, which makes investigating this mechanism rather difficult [34]. However, it should be carefully considered that Y-27632 could also have negative effects on the cryopreservation of stem cells, such as a reduced expansion and cell survival as observed for cord blood stem cells [35] or morphological changes linked with initiating differentiation observed for mesenchymal stem cells [36]. In our setup, no Y-27632 was added and good results were obtained.

As inherent differences between hESC lines [37] exist, we investigated the effect of our optimized cryopreservation protocol on 5 hESC lines. Protocol 2 with 5\% DMSO and 5\% HES led to the highest recovery rates, ranging from $45.5 \%$ for the $\mathrm{H} 1$ cell line to $168.2 \%$ for the $\mathrm{H} 9$ cell line. It might be atypical to obtain a recovery rate higher than $100 \%$, but this simply is due to the fact that the hESC colonies are processed into smaller clumps during the freezing process, which can subsequently give rise to a higher number of new colonies at thawing.

Not only the recovery ratio is important when studying hESC cryopreservation, also their undifferentiated status, expansive behavior, and expression profile should be examined. Analysis of the previously frozen hESC colonies reveal that they still express high amounts of SSEA-3, SSEA4, TRA 1-60, TRA 1-81, Oct4, and ALP, all characteristic markers of pluripotent hESCs. The expansive capacity of the different frozen samples is clearly visible in the 3D bar graph. The condition with the overall best recovery ratio, elicits the best expansive profile. Less than $5 \%$ differentiation occurred in our best condition. Hence, the use of 5\% DMSO in combination with $5 \%$ HES not only results in a high recovery ratio after freezing, but also allows good cell expansion, which is of key importance for future applications. Real-time PCR analysis of the EBs showed a decrease in Oct4 and Nanog expression and an increase in expression of mesodermal, endodermal, and ectodermal markers, indicating their pluripotent cell nature and capacity to differentiate to the 3 germ layers.

On the basis of our results, we conclude that protocol 2 with 5\% DMSO and 5\% HES is ideal for slow freezing of hESCs. In this protocol, the hESCs are detached with collagenase IV, treated with the cell dissociation solution to obtain a more uniform size distribution of the colonies, and slowly frozen in HM medium with only 5\% DMSO and 5\% HES. Rapid thawing and equilibration into $0.1 \mathrm{M}$ sucroseHM medium resulted in a good colony startup and expansion profile. In addition to a minimal amount of synthetic serum, no additional substances (such as Y-27632) were added and a nonenzymatic, animal-free dissociative solution was used. As HES and the cell dissociation solution are quite cheap when compared with other possible additions (such as Y-27632), we suggest that this is an economically feasible protocol for the freezing of large amounts of hESCs for hESC banking.

\section{Acknowledgments}

This study was conducted as part of a Ph.D. project, funded by BOF (BOF08/24J/063). The authors express gratitude to Prof. Dr. Karen Sermon and Dr. Ileana Mateizel for donating the VUB01 hESC line and to Prof. Dr. Petra De Sutter and Dr. Björn Heindryckx for donating the UGent2 hESC line. The authors also thank Johanna Aernoudt and Leen Pieters, lab assistants, for their practical aid in realizing this work.

\section{Author Disclosure Statement}

There are no potential conflicts of interest.

\section{References}

1. Thomson JA, J Itskovitz-Eldor, SS Shapiro, MA Waknitz, JJ Swiergiel, VS Marshall and JM Jones. (1998). Embryonic stem cell lines derived from human blastocysts. Science 282:1145-1147.

2. Mountford JC. (2008). Human embryonic stem cells: origins, characteristics and potential for regenerative therapy. Transfus Med 18:1-12.

3. Battey JF. (2007). Stem cells: current challenges and future promise. Dev Dyn 236:3193-3198.

4. Li T, QY Mai, J Gao and CQ Zhou. (2010). Cryopreservation of human embryonic stem cells with a new bulk vitrification method. Biol Reprod 82:848-853.

5. Li T, CQ Zhou, CX Liu, QY Mai and GL Zhuang. (2008). Bulk vitrification of human embryonic stem cells. Hum Reprod 23:358-364.

6. Li Y TJ, Li L. (2008). Comparison of three methods for cryopreservation of human embryonic stem cells. Fertil Steril 93:999-1005.

7. Richards M, CY Fong, S Tan, WK Chan and A Bongso. (2004). An efficient and safe xeno-free cryopreservation method for the storage of human embryonic stem cells. Stem Cells 22:779-789.

8. Meryman HT. (2007). Cryopreservation of living cells: principles and practice. Transfusion 47:935-945.

9. Blitterswijk CV. (2008). Tissue engineering. In: Academic Press Series in Biomedical Engineering. J Bronzino, ed. Elsevier, London (UK), p363-403.

10. Karlsson JOM and M Toner. (1996). Long-term storage of tissues by cryopreservation: critical issues. Biomaterials 17:243-256.

11. Holm F, S Strom, J Inzunza, D Baker, AM Stromberg, B Rozell, A Feki, R Bergstrom and O Hovatta. (2010). An effective serum- and xeno-free chemically defined freezing procedure for human embryonic and induced pluripotent stem cells. Hum Reprod 25:1271-1279.

12. Ha SY, BC Jee, CS Suh, HS Kim, SK Oh, SH Kim and SY Moon. (2005). Cryopreservation of human embryonic stem cells without the use of a programmable freezer. Hum Reprod 20:1779-1785.

13. Wu CF, HC Tsung, WJ Zhang, Y Wang, JH Lu, ZY Tang, YP Kuang, W Jin, L Cui, W Liu and YL Cao. (2005). Improved cryopreservation of human embryonic stem cells with trehalose. Reprod Biomed Online 11:733-739.

14. Fleming KK and A Hubel. (2006). Cryopreservation of hematopoietic and non-hematopoietic stem cells. Transfus Apheresis Sci 34:309-315.

15. Ji L, JJ de Pablo and SP Palecek. (2004). Cryopreservation of adherent human embryonic stem cells. Biotechnol Bioeng 88:299-312. 
16. Li XY, R Krawetz, SY Liu, GL Meng and DE Rancourt. (2009). ROCK inhibitor improves survival of cryopreserved serum/feeder-free single human embryonic stem cells. Hum Reprod 24:580-589.

17. Martin-Ibanez R, O Hovatta and JM Canals. (2009). Are stem cells inherently more vulnerable to cryopreservationinduced apoptosis compared with ordinary somatic cells? Reply. Hum Reprod 24:492-493.

18. Martin-Ibanez R, C Unger, A Stromberg, D Baker, JM Canals and O Hovatta. (2008). Novel cryopreservation method for dissociated human embryonic stem cells in the presence of a ROCK inhibitor. Hum Reprod 23:2744-2754.

19. Mollamohammadi S, A Taei, M Pakzad, M Totonchi, A Seifinejad, N Masoudi and H Baharvand. (2009). A simple and efficient cryopreservation method for feeder-free dissociated human induced pluripotent stem cells and human embryonic stem cells. Hum Reprod 24:2468-2476.

20. Watanabe K, M Ueno, D Kamiya, A Nishiyama, M Matsumura, T Wataya, JB Takahashi, S Nishikawa, K Muguruma and Y Sasai. (2007). A ROCK inhibitor permits survival of dissociated human embryonic stem cells. Nat Biotechnol 25:681-686.

21. Clapisson G, C Salinas, Malacher P, Michallet M, Philip I, Philip T. (1999). Cryopreservation with hydroxyethylstarch (HES) + dimethylsulfoxide (DMSO) gives better results than DMSO alone. Electron J Oncol 1:97-102.

22. Stiff PJ. (1995). Cryopreservation of Hematopoietic Stem Cells. American Association of Blood Banks, Tampa, FL.

23. Hubel A. (1997). Parameters of cell freezing: implications for the cryopreservation of stem cells. Transfus Med Rev 11:224-233.

24. Brockbank KGM, JC Covault, MJ. Taylor (2007). Cryopreservation Guide. Thermo Scientific Publications. www.thormofisher.com, 1-10.

25. Karlsson JOM. (2002). Cryopreservation: freezing and vitrification. Science 296:655-656.

26. Mateizel I, N De Temmerman, U Ullmann, G Cauffman, K Sermon, H Van de Velde, M De Rycke, E Degreef, P Devroey, I Liebaers and A Van Steirteghem. (2006). Derivation of human embryonic stem cell lines from embryos obtained after IVF and after PGD for monogenic disorders. Hum Reprod 21:503-511.

27. O'Leary T, B Heindryckx, S Lierman, M Van der Jeught, B Menten, D Deforce, R Cornelissen, SCD Lopes and P De Sutter. The Influence of early embryo traits on human embryonic stem cell derivation efficiency. Stem Cells Dev 20:785-793.

28. Richards M, CY Fong, S Tan, WK Chan and A Bongso. (2005). An efficient and safe xeno-free cryopreservation method for the storage of human embryonic stem cells. Stem Cells 23:604-604.
29. Taylor JR. (1997). An Introduction to Error Analysis. University Science Books, Sausalito, CA.

30. Tedder RS, MA Zuckerman, AH Goldstone, AE Hawkins, A Fielding, EM Briggs, D Irwin, S Blair, AM Gorman, et al. (1995). Hepatitis-B transmission from contaminated cryopreservation tank. Lancet 346:137-140.

31. T'Joen V, Declercg $H$, and Cornelissen M. Expansion of human embryonic stem cells: a comparative study. Cell Prolif (In press).

32. Baharvand H, GH Salekdeh, A Taei and S Mollamohammadi. (2006). An efficient and easy-to-use cryopreservation protocol for human ES and iPS cells. Nat Protoc 5:588-594.

33. Lee JY LJ, Kim DK, Yoon TK, Chung HM, Lee DR. (2010). High concentration of synthetic serum, stepwise equilibration and slow cooling as an efficient technique for large-scale cryopreservation of human embryonic stem cells. Fertil Steril 93:976-985.

34. Krawetz RJ, X Li and DE Rancourt. (2009). Human embryonic stem cells: caught between a ROCK inhibitor and a hard place. Bioessays 31:336-343.

35. Bueno C, R Montes and P Menendez. (2010). The ROCK inhibitor Y-27632 negatively affects the expansion/survival of both fresh and cryopreserved cord blood-derived CD34+ hematopoietic progenitor cells: Y-27632 negatively affects the expansion/survival of CD34+ HSPCs. Stem Cell Rev 6:215-223.

36. Heng BC. (2009). Effect of Rho-associated kinase (ROCK) inhibitor Y-27632 on the post-thaw viability of cryopreserved human bone marrow-derived mesenchymal stem cells. Tissue Cell 41:376-380.

37. Allegrucci C and LE Young. (2007). Differences between human embryonic stem cell lines. Hum Reprod Update 13:103-120.

Address correspondence to: Veronique T'Joen, MBS

Tissue Engineering Group Department of Basic Medical Science Faculty of Medicine and Health Science Ghent University-UGent De Pintelaan 1856 63 9000 Gent Belgium

E-mail: veronique.tjoen@ugent.be

Received for publication April 18, 2011

Accepted after revision June 2, 2011

Prepublished on Liebert Instant Online June 3, 2011 